\title{
Data Analysis Techniques for Fan Performance in Highly- Distorted Flows from Boundary Layer Ingesting Inlets
}

\author{
Larry W. Hardin, ${ }^{1}$ and William T. Cousins. ${ }^{2}$ \\ United Technologies Research Center, 411 Silver Lane, East Hartford, CT, 06118, USA \\ and \\ John D. Wolter ${ }^{3}$, David J. Arend ${ }^{4}$, and Stefanie M. Hirt ${ }^{5}$ \\ NASA Glenn Research Center, Cleveland, Ohio, 44135, USA
}

\begin{abstract}
The design of a unique distortion-tolerant fan for a high-bypass ratio boundary-layer ingesting propulsion system has been completed and a rig constructed and tested in the NASA Glenn 8'x6' wind tunnel. Processing the data from the experiment presented some interesting challenges because of the complexity of the experimental setup and the flow through the test rig. The experiment was run in three phases, each of which employed a unique complement of inlet throat and fan face instrumentation to avoid the blockage that would have resulted from simultaneously installing all of the rakes. The measurement from the individual test points were subsequently combined to compute the overall stage performance. A CFD model of the experiment was used to gain understanding of the flow field and to test some of the techniques proposed for interpolating and extrapolating the measurements into regions where measurements were not made. This capability became extremely useful when it was discovered that there was an unexpected total temperature distortion in the tunnel. The CFD model was modified by inserting a total temperature profile at the upstream boundary that mimicked the measured distortion where measurements were available and that CFD solution was used to investigate methods to infer the complete total temperature field at the fan face.
\end{abstract}

\section{Nomenclature}

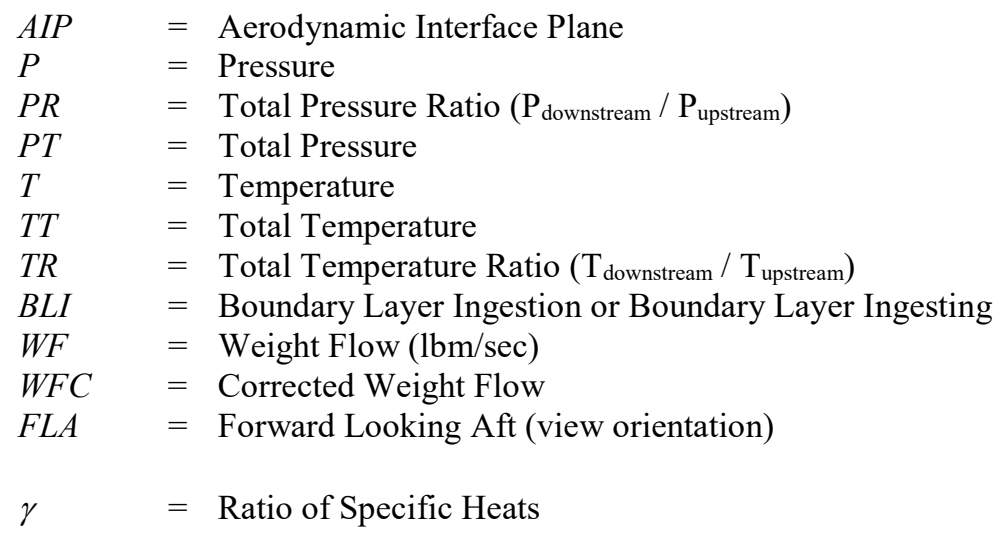

\footnotetext{
${ }^{1}$ Staff Engineer, Aerodynamics, Senior Member.

${ }^{2}$ Fellow, Thermal Fluid Sciences, AIAA Associate Fellow.

${ }^{3}$ Aerospace Engineer, Inlets and Nozzles Branch, Associate Fellow.

${ }^{4}$ Aerospace Engineer, Inlets and Nozzles Branch, Associate Fellow.

${ }^{5}$ Aerospace Engineer, Inlets and Nozzles Branch, Senior Member.
} 


\section{Introduction}

$\mathrm{T}_{\mathrm{s}}^{\mathrm{H}}$ HE foundations of Boundary Layer Ingesting Propulsion can be found in the paper by Smith [1] which described the ingesting of the boundary layer of a body of revolution by a pusher propeller. The resulting radial velocity profile did not produce harmonic excitation of the propulsor blades resulting in a relatively simple structural design problem. The Robust Design of Embedded Engine Systems (RDEES) contract targeted a blended wing body with top-mounted engines. An aircraft level system study was conducted using a Boeing Hybrid Wing Body (HWB) design which is described in Ref. [2] and Ref. [3]. Boeing kindly provided a CFD model of that configuration which was used to estimate the flow profile that would enter the inlets at various mounting locations. A Numerical Propulsion System Simulation (NPSS) of an advanced geared turbofan provided by Pratt \& Whitney and modified to account for Boundary Layer Ingestion, yielded estimates of fuel consumption for various engine configurations. The system study which is described in [4] predicted that the best performance would be attained with five geared turbofan engines embedded in the aft portion of the airframe as shown in Figure 1. This configuration results in a highly distorted total pressure profile entering the inlet, leading to significant aerodynamic and structural challenges.

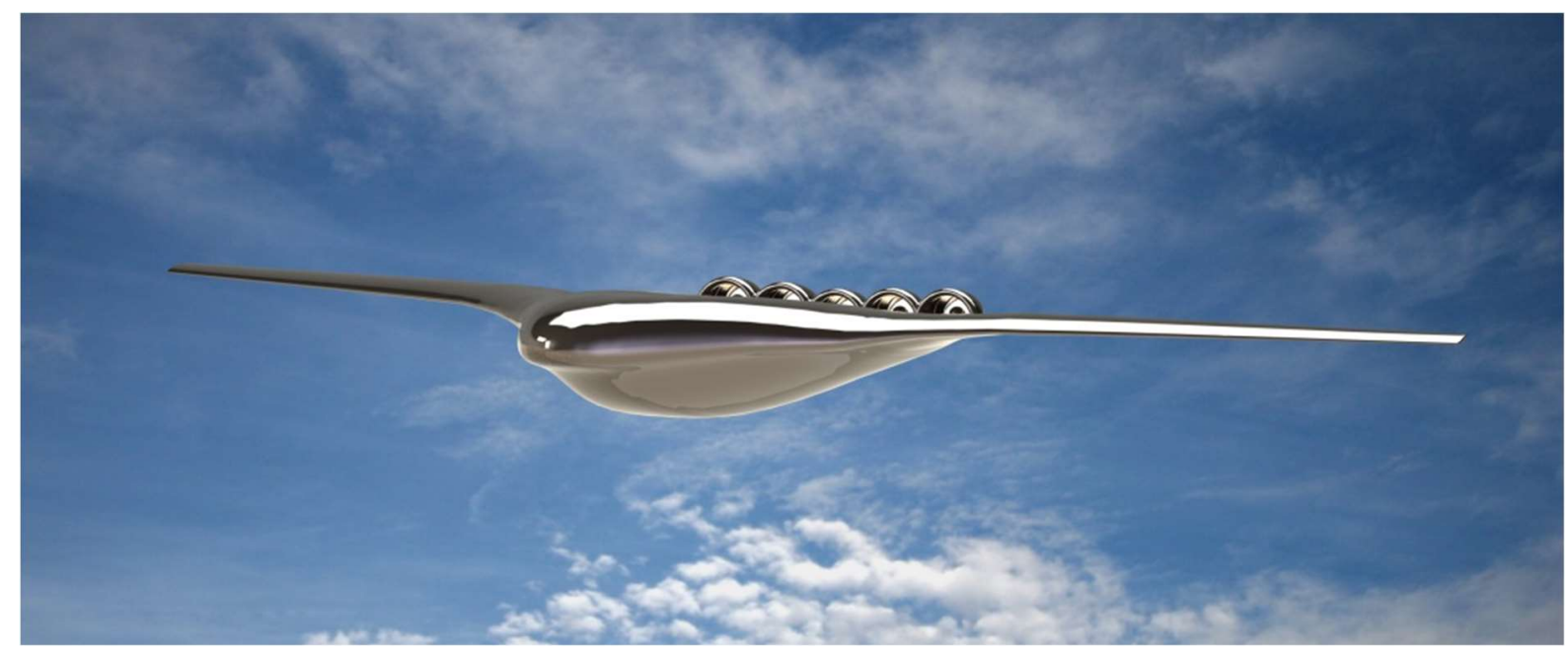

Figure 1. Basic Configuration Studied for Robust Design of Embedded Engine Systems

The system study concluded that a low loss inlet that required minimal bleed for flow control would be necessary to achieve the desired fuel burn benefits. A parametric analysis of the inlet system was performed [5] and the results were folded into the system study to compute the overall benefits of the coupled airframe, inlet, and engine configuration. It was not possible to fully remove the distortion because doing so with flow control would require more bleed air than could be extracted from the engines without a significant impact on their performance and attempting to simply mix out the distortion would entail unacceptable mixing losses and a long, heavy inlet that would compromise overall performance. The only option available was to design the fan to tolerate a level of distortion that normally would not be encountered except in the case of a military fighter aircraft executing extreme maneuvers. The process by which this Distortion Tolerant Fan (DTF) was designed is described in [9]. In parallel with the design and fabrication of the inlet and fan stage at UTRC, engineers at NASA Glenn Research Center (GRC) designed and fabricated the hardware necessary to produce an inlet total pressure profile representative of that expected during cruise for the aircraft [6]. Because of the extreme circumferential distortion, it was necessary to design and fabricate rotating rake arrays that were installed at the Aerodynamic Interface Plane (AIP) and the exit plane of the Fan Exit Guide Vanes (FEGV). These rotating rake arrays were designed by the engineers at GRC [7]. One of the issues with experimental measurements is that it is almost never possible to measure everything one would wish to know, and even if that were possible, the blockage created by the instrumentation would be such that the results would not represent the performance of the rig with the instrumentation removed. GRC engineers optimized the placement of the instrumentation using a CFD solution of the flow through the test rig to obtain the greatest accuracy with the instrumentation that could be installed [7]. The hardware was assembled into the wind tunnel and the test program was executed at the end of 2016. Preliminary results [8] [9] show performance that is a reasonable match to the pre-test predictions and far more stall margin that had been expected. 


\section{Three Phases of AIP Measurements}

A key issue facing the instrumentation designers was that the number of rakes and probes required to map the distortion at the Aerodynamic Interface Plane (AIP) would cause blockage that would adversely affect the measurement of the fan performance. This concern was addressed in the RDEES experiment by running the experiment in three phases with the differences being in the instrumentation installed at the AIP. These phases are illustrated in Figure 2.

AIP Phase I

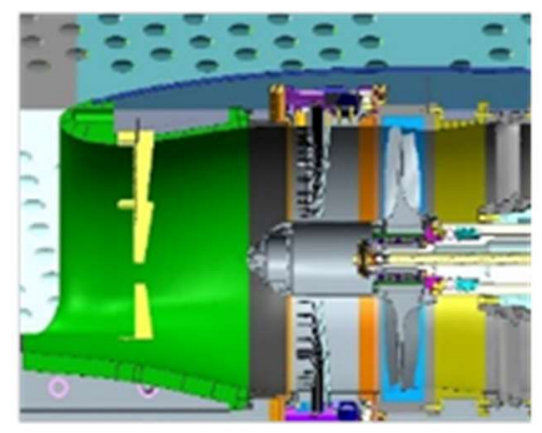

- Inlet Throat Rakes

- Extended Spinner

- AlP Spool

- AIP Rakes
AIP Phase II

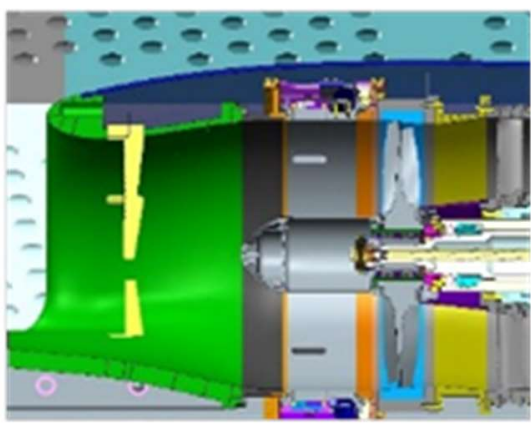

- Inlet Throat Rakes

- Extended Spinner

- AlP Spool

- No AIP Rakes
AIP Phase III

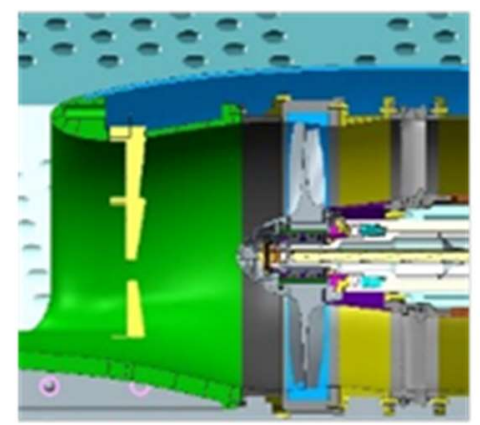

- Inlet Throat Rakes

- Conventional Spinner

- No AIP Spool

- No AlP Rakes

Figure 2. Three Phase Approach to Acquiring Fan Performance Data

For all three phases of the testing, the Fan Exit Rotating Rake Array (FERRA) were installed. The inlet throat rakes were optional for all three phases. Phase I instrumentation included a large number of probes at the AIP to characterize the flow entering the fan. The AIP probes consist of Kiel heads for measuring total pressure, Kiel heads with embedded thermocouples for measuring total temperature, and cobra probes which yielded total pressure, static pressure, and flow yaw angle. These probes were mounted onto eight rakes which were installed into a rotating spool so that they could be traversed through the distortion in fine increments. Installation of the AIP spool required shifting the inlet forward and installing an extended spinner. The modified spacing necessarily impacts the manner in which the inlet and fan interact. Phase II was run with the AIP spool piece installed, but with the AIP rakes removed. This configuration mimics the Phase III configuration with the only difference being the different separation between the fan and the inlet. Thus, differences between the inlet throat and Fan Exit Rotating Rake Array (FERRA) measurements with and without the spool piece installed (i.e. Phase II and Phase III) provide a measure of the impact of the change in spacing between the fan and the inlet. To date, this analysis has not been conducted.

The weight flow (measured at the FERRA plane) corrected to free stream conditions is plotted as a function of nozzle position in Figure 3 at 100\% corrected RPM for Phase I and Phase III. If the AIP rakes had produced no loss and the introduction of the AIP spool had no influence on the inlet/fan interaction, the two data sets would be very similar. The fact that Phase I produces a lower weight flow than Phase III for the same nozzle position indicates that the AIP rakes introduce significant blockage. 


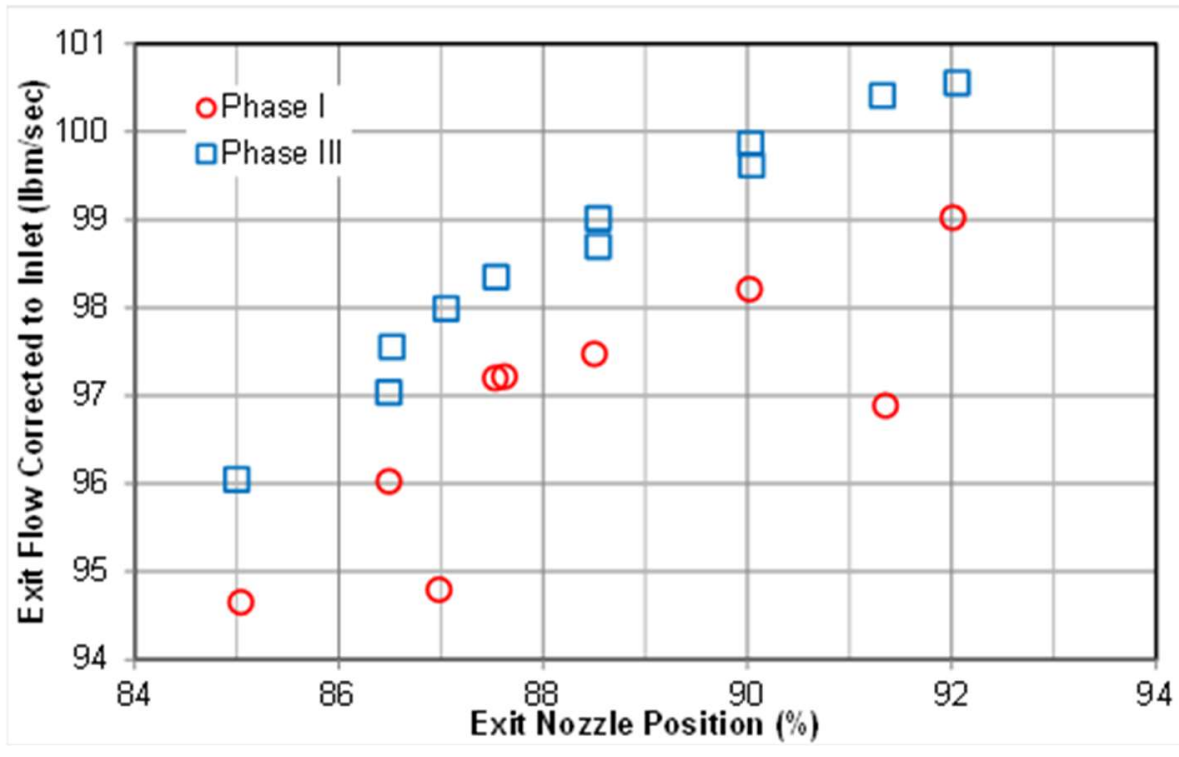

Figure 3. Corrected Flow as a Function of Exit Nozzle Position

It was postulated that the inlet losses should be a function of the corrected flow. To determine whether this was true, the ratio of the mass-averaged total pressure at the AIP to freestream total pressure (outside the imposed boundary layer profile) is plotted as a function of the weight flow corrected to free stream (reference) conditions in Figure 4. The red circles represent the surveys conducted at 100 percent corrected RPM and the blue squares represent the surveys conducted at a nozzle setting of 87.5 percent open, which is a notional operating line. Note that the fan alone cannot have an operating line, but this nozzle setting approximates what was assumed to be the operating line during the design process. It is important to realize that this recovery factor is NOT the inlet pressure recovery because it is referenced to a single total pressure that is measured outside of the induced distortion and thus includes both the inlet loss as well as the imposed distortion. The solid green line represents a linear least squares fit to the measured data and the dashed green lines represent an error band of \pm 0.00025 relative to that fit. Most of the data lie within that error band. This error band is rather arbitrary and will be used to illustrate the error in the stage pressure ratio and stage efficiency that might result from the data scatter shown.

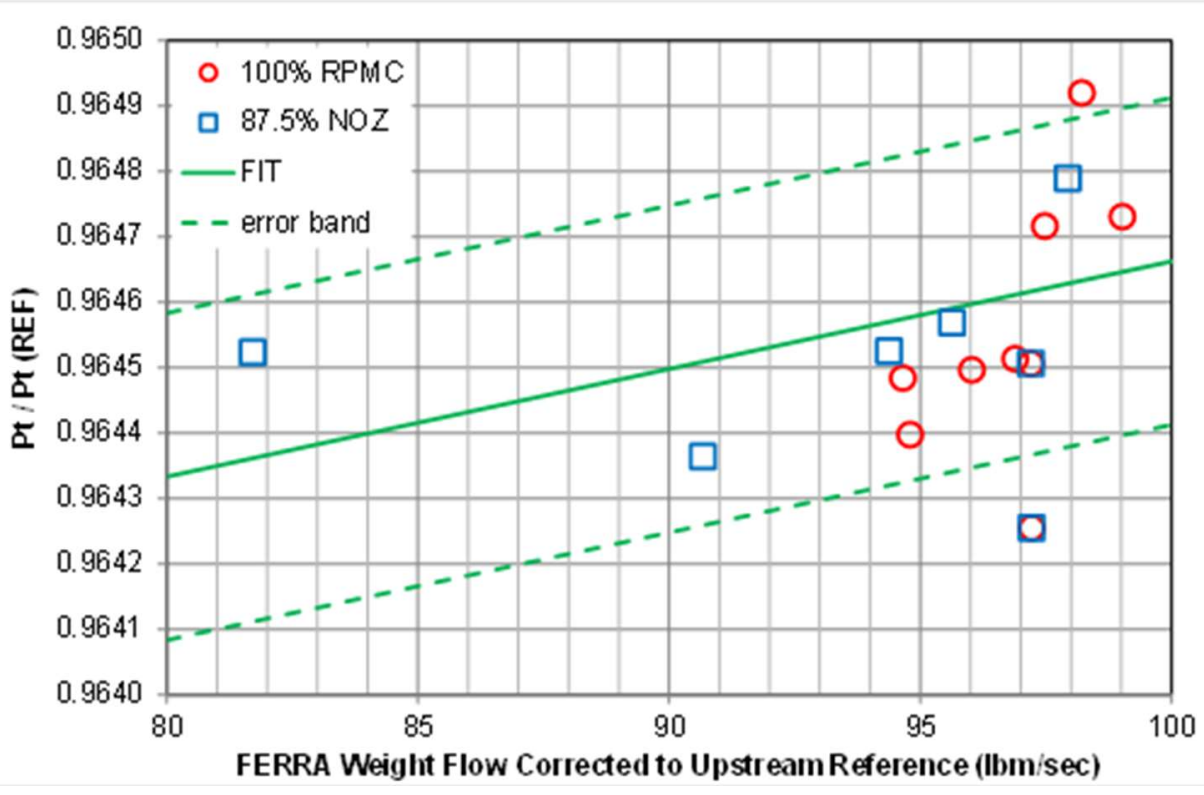

Figure 4. Total Pressure Recovery as a Function of Weight Flow 
In a similar fashion, Figure 5 presents the ratio of the mass-averaged total temperature at the AIP plane to the reference free stream total temperature. The error band used for total temperature is only \pm 0.00015 .

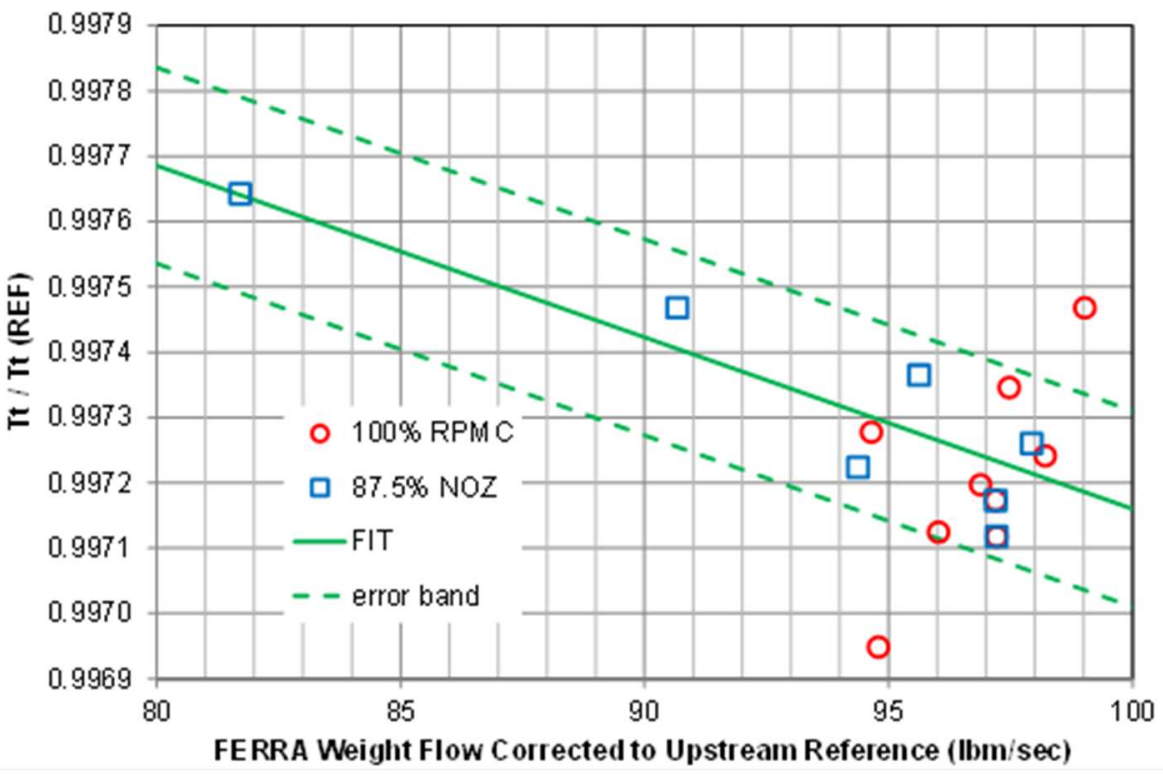

Figure 5. Total Temperature Recovery as a Function of Weight Flow

The pressure ratio and efficiency of the fan stage can be calculated from the Phase III surveys by using the curve fits for the upstream total pressure and total temperature applied to the weight flow computed at the FERRA plane and corrected to the upstream reference to obtain the ratios referenced in the curve fits. Multiplying these ratios by the reference total pressure and total temperature for each Phase III survey produces an estimate of the dimensional total pressure and total temperature at the AIP. The error bands provide estimates of the highest and lowest values that might be expected and can be used to create error bars on the plotted pressure ratio and efficiency estimates. The stage pressure ratio as a function of the weight flow corrected to the AIP conditions is shown in Figure 6.

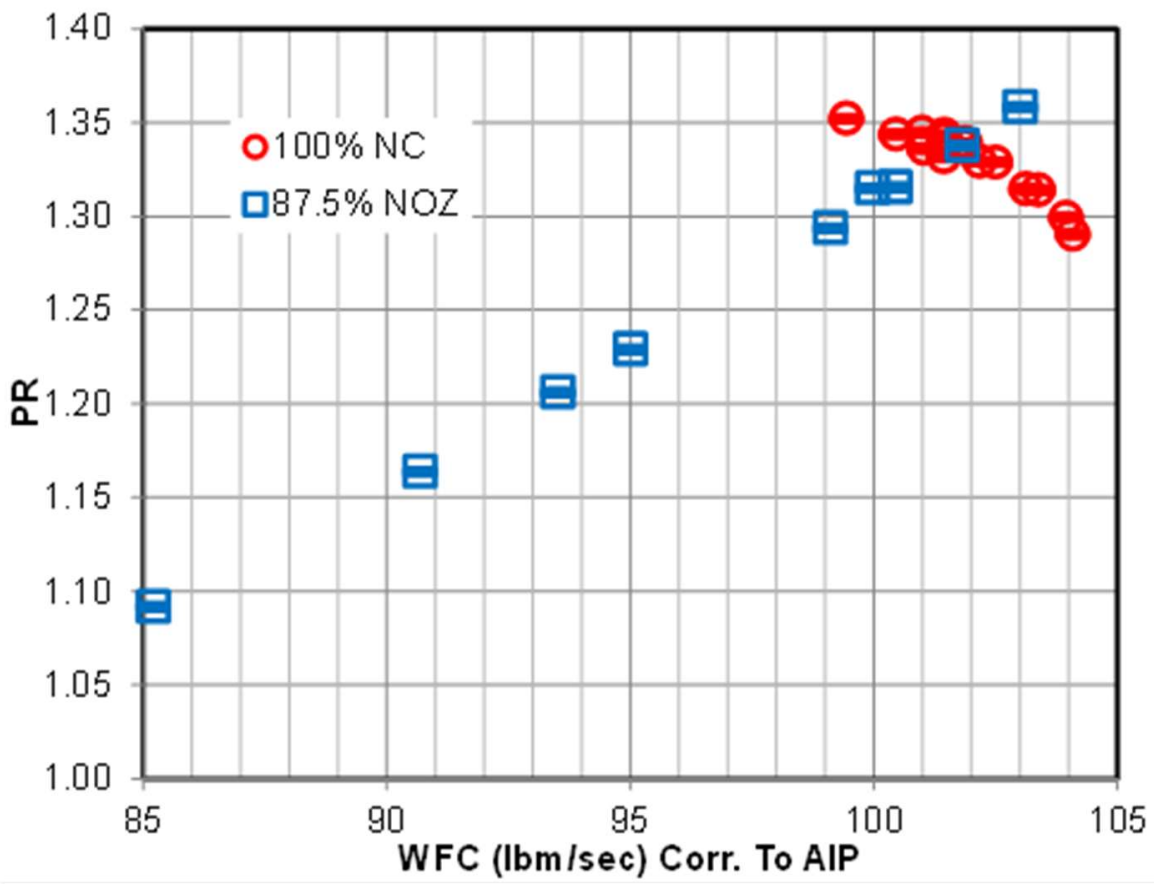

Figure 6. Stage Pressure Ratio as a Function of Corrected Weight Flow 
The error bars are so small that they are contained entirely within the data symbol. It must be admitted that these error bars only represent the likely error associated with the process of fitting the Phase I data to the corrected flow. Other error sources are possible, but these have not been quantified.

The adiabatic efficiency is computed according to Eq.( 1 ). In computing the error bars on the efficiency, the upper error band on the total pressure must be paired with the lower error band on the total temperature and viceversa.

$$
\eta=\frac{P R^{(\gamma-1)} / \gamma_{-1}}{T R-1}
$$

The stage efficiency is plotted as a function of weight flow corrected to the AIP conditions in Figure 7. The error bars are slightly larger, but still quite reasonable. Maximum efficiency does not occur at $100 \%$ corrected speed; instead, it occurs at approximately $87.5 \%$ RPMC. The maximum efficiency on the $100 \%$ RPMC curve occurs for a nozzle setting of $87.2 \%$ open, which suggests that the choice of $87.5 \%$ open as a notional operating line is not unreasonable.

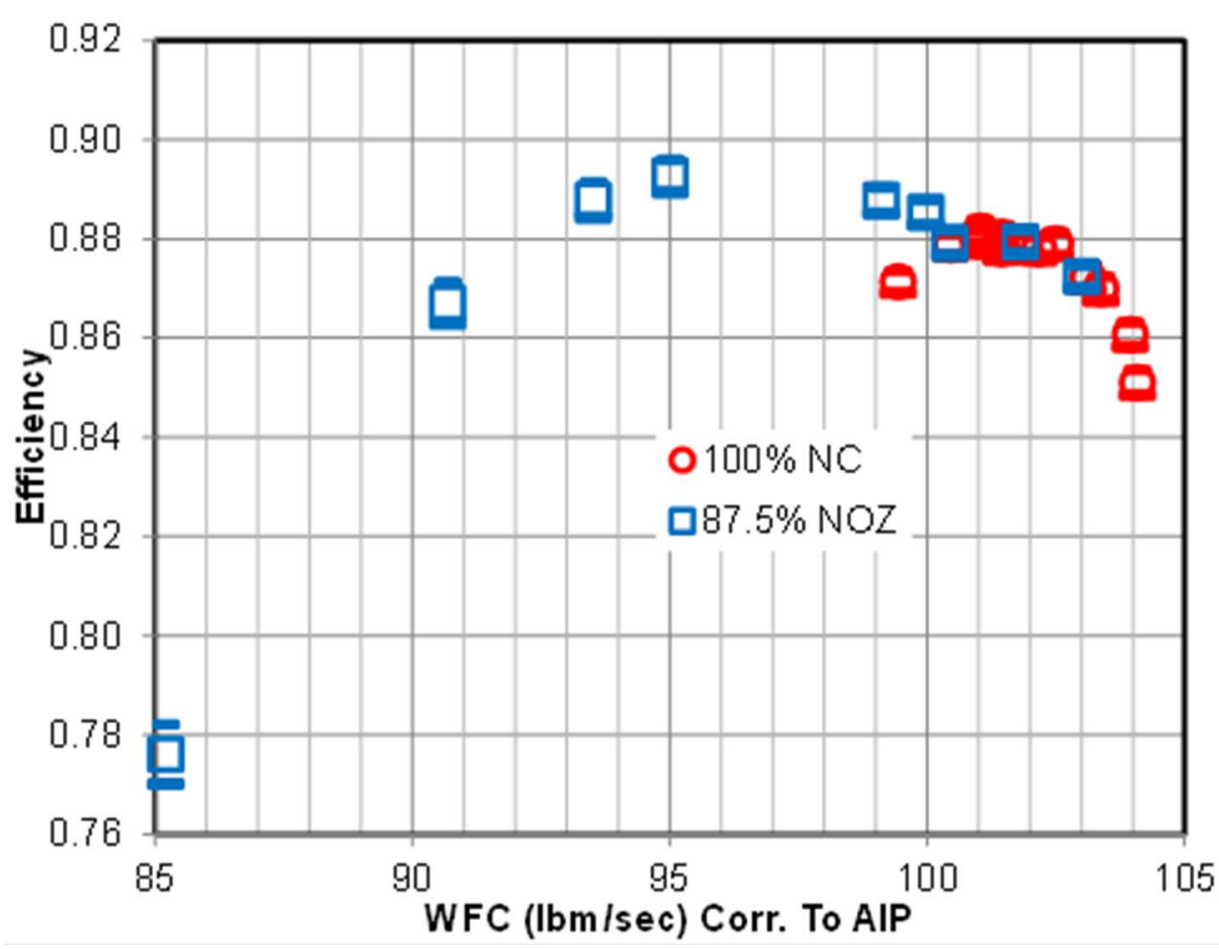

Figure 7. Stage Efficiency as a Function of Corrected Weight Flow

\section{Reconstructing the Total Temperature Profile at the AIP}

The original design of the experiment assumed a uniform total temperature upstream of the inlet. During the test, it was found that there was a gradient in total temperature such that the mass averaged total temperature at the Aerodynamic Interface Plane (AIP) was lower than the free stream value. Anticipating the uniform temperature, the AIP temperature instrumentation was sparse, consisting of only two rings of total temperature probes. In order to explore a means of recreating the temperature profile at the AIP, a CFD case was run with an upstream boundary condition which produced temperatures at the AIP that approximated the measured data. Based on a working hypothesis that the distortion was the result of the outer walls of the tunnel being cold, a two dimensional profile was applied at the upstream boundary condition of the CFD simulation. The total temperature distribution thus 
obtained at the AIP is shown in Figure 8 with the two rings of measuring locations being indicated by the dashed lines. This CFD solution was sampled to provide the same data granularity as the measured data and used to test the proposed processing techniques.

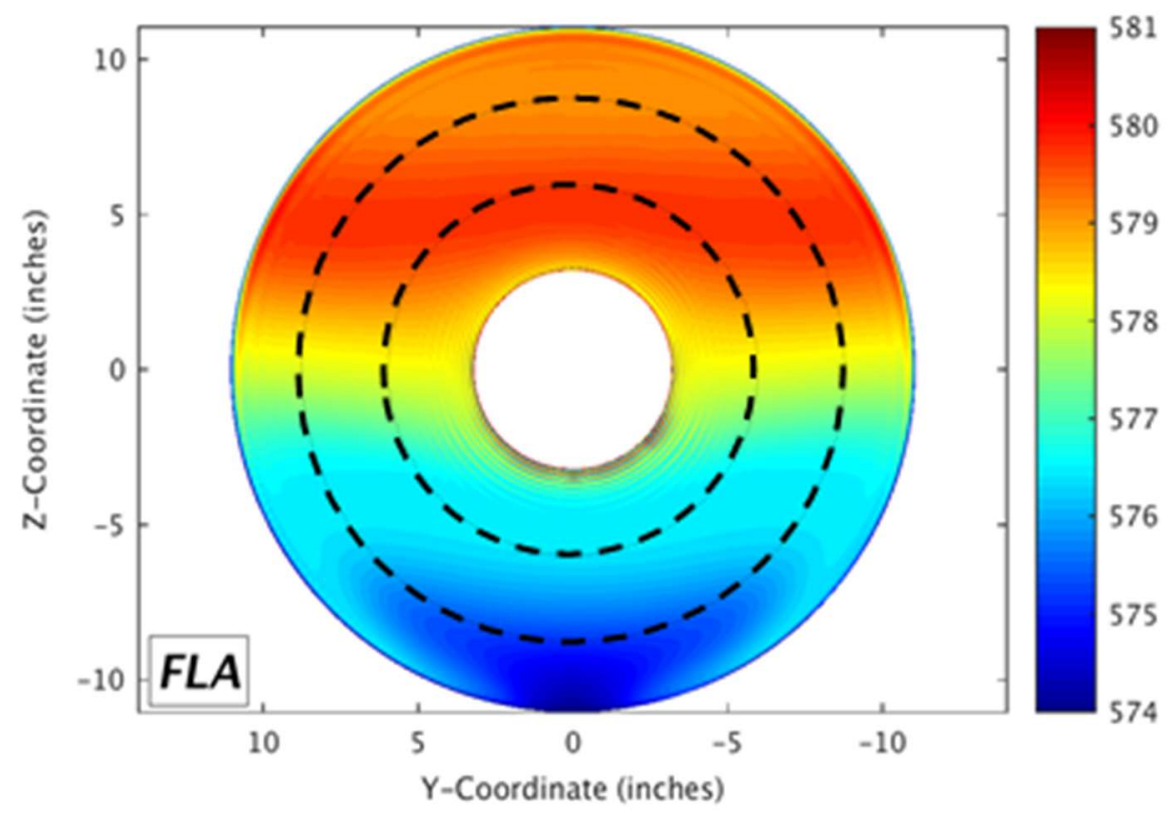

Figure 8. Total Temperature at AIP from CFD Solution with Non-uniform Upstream Profile

Noting that both total pressure and total temperature are convected along streamlines, it was theorized that it might be possible to develop a correlation between the measured total temperature and measured total pressures on the two rings shown above that could be used to extend the measured temperatures into regions where the temperature had not been measured but the total pressure had been measured. This relationship should be valid wherever there is no heat addition or pressure loss along those streamlines. Those conditions are met except for the fluid in the boundary layer. Figure 9 demonstrates that the relationship between total temperature and total pressure is usable for this purpose and illustrates the resulting total temperatures in the lower part of the AIP.

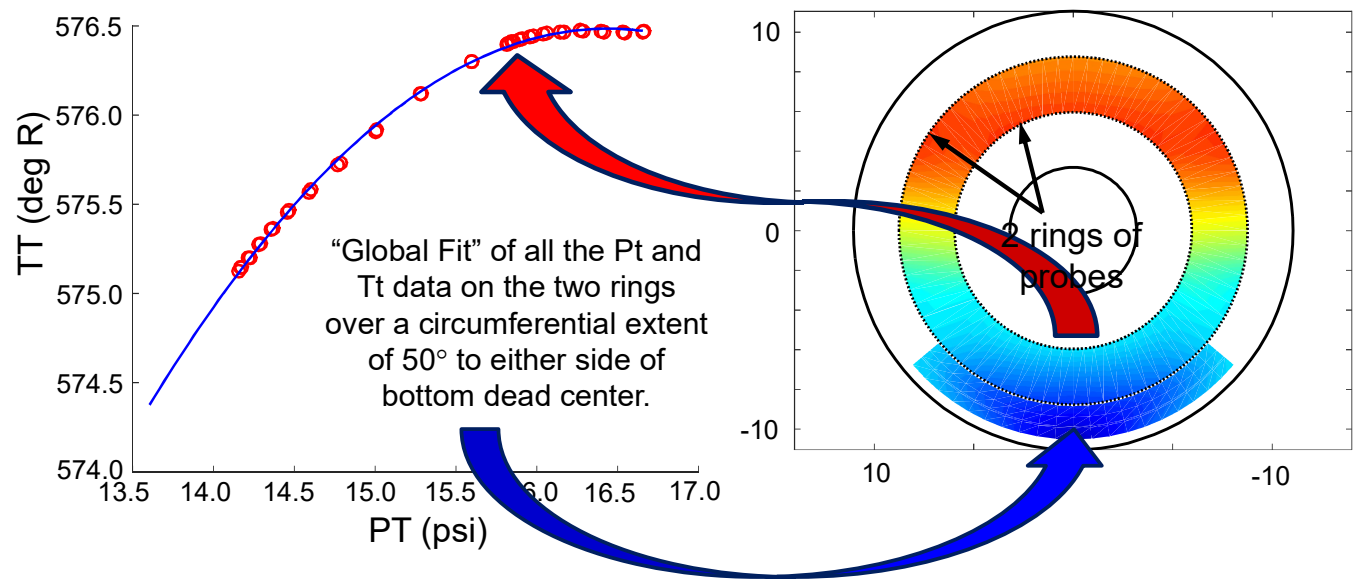

Fills in Bottom Sector

Figure 9. Using a Curve Fit of Total Temperature as a Function of Total Pressure to Fill in the Lower Part of the AIP (CFD solution shown) 
The region between the two rings where total temperature was actually measured was interpolated by triangularizing the region and applying Barycentric interpolation. The arc sector outside the outer ring in the bottom region was extrapolated using the previously described fit of the total temperature measurements on the rings as a function of total pressure. The white region at the bottom of the AIP is the part of the flow that is the boundary layer. Figure 10 further shows that the estimated total temperatures in that region are a reasonable approximation of the full CFD solution.

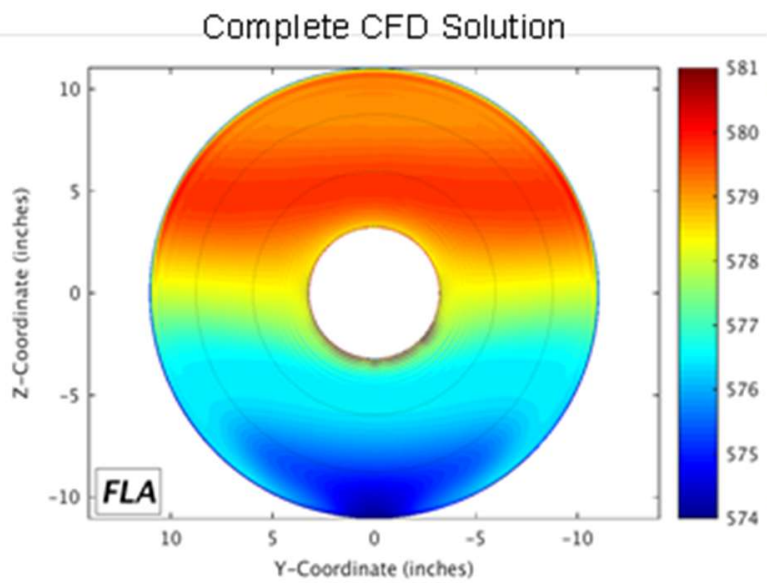

\section{Constructed from "Measured" Data}

Fit Pt and Tt at each circumferential location to fill in Tt at Pt Rake Points

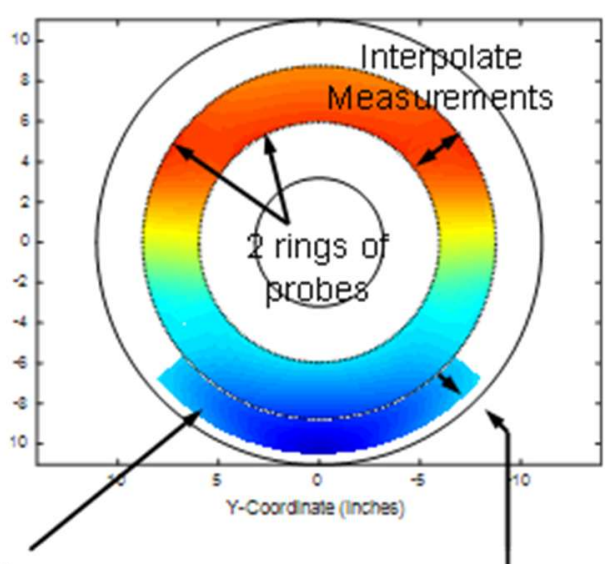

Technique does not work for points in the boundary layer

Figure 10. Complete CFD Solution Compared to Reconstruction from "Measured" (i.e. Sampled CFD) Data

For the CFD results, there is little scatter of the "measured" points; however, it must be recognized that the imposed total pressure and total temperature profiles are two-dimensional at the upstream location where boundary conditions are imposed and may not reflect reality. Figure 11 shows the equivalent relationship from one of the experimental data points. Although there is some scatter, there is a strong correlation that permits creation of an interpolating function shown by the blue curve.

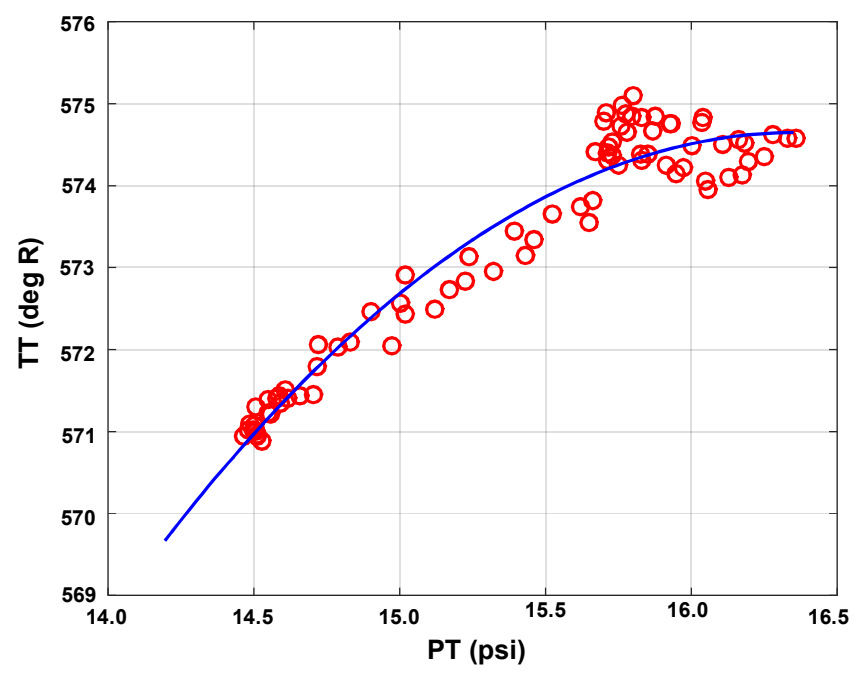

Figure 11. Total Temperature as a Function of Total Pressure from Experimental Data Point

This plot also shows a temperature variation of almost 4 degrees $F$ from the lowest measuring point to the uppermost measuring point. The temperature profile required to reproduce this variation in the CFD study resulted in a 
temperature variation of 7 degrees from the bottom of the AIP to the top of the AIP. The resultant difference between the mass-flow averaged total temperature and the free stream value was on the order of 3 degrees for the CFD solution, indicating that it was critical to devise a method to capture the impact of the total temperature distortion.

It was assumed that the two dimensional nature of the upstream profile might persist to the AIP; although, it might be shifted in some manner by the passage of the flow over the pre-entry diffusion ramp upstream of the inlet and around the inlet lip. Plotting the total temperature at the AIP along with that external to the nacelle (Figure 12) supports this assumption. The experiment included five total temperature probes shown by the five white crosses which can be combined with the outer ring of AIP total temperature probes to provide data for a fit that will fill in the upper portion of the AIP.

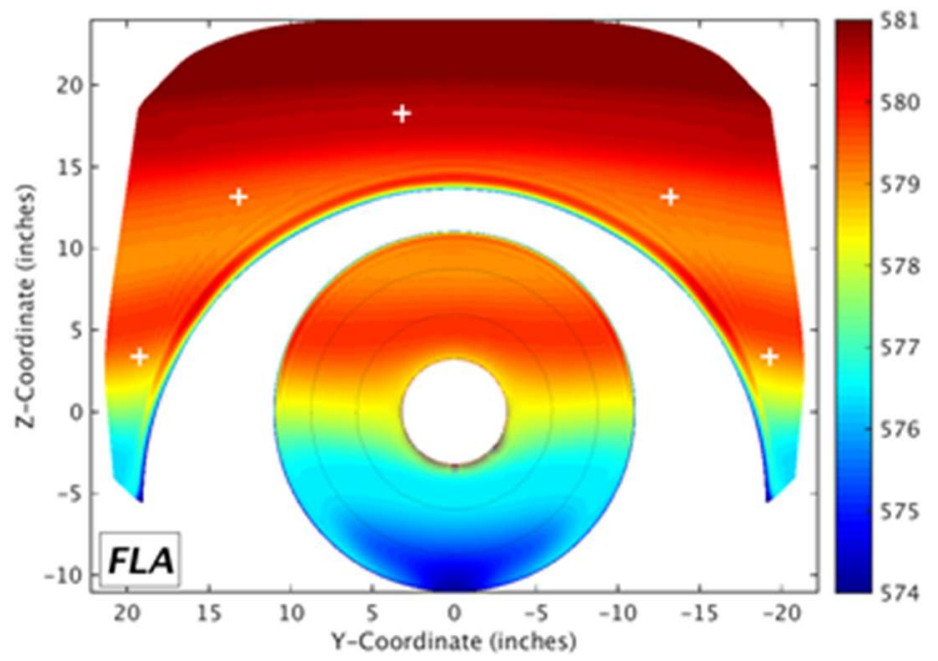

Figure 12. Total Temperature Distribution Inside and Outside the Nacelle at the AIP

Extracting the equivalent of the measured data from the CFD solution and plotting as a function of the Zcoordinate provides an interpolating polynomial fit as shown in Figure 13. The fit shown by the green line is limited to the Z-coordinates that lie inside the nacelle.

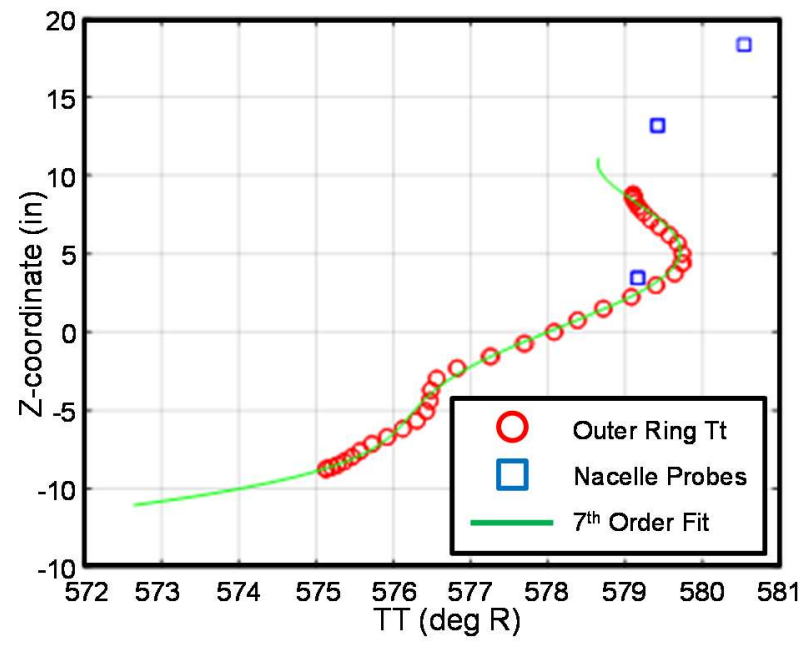

Figure 13. Curve Fit of Sampled CFD Results Inside and Outside Nacelle at AIP 
Figure 14 compares the reconstruction of the total temperature contours from the sampled CFD solution to the full solution. The differences are relatively minor.
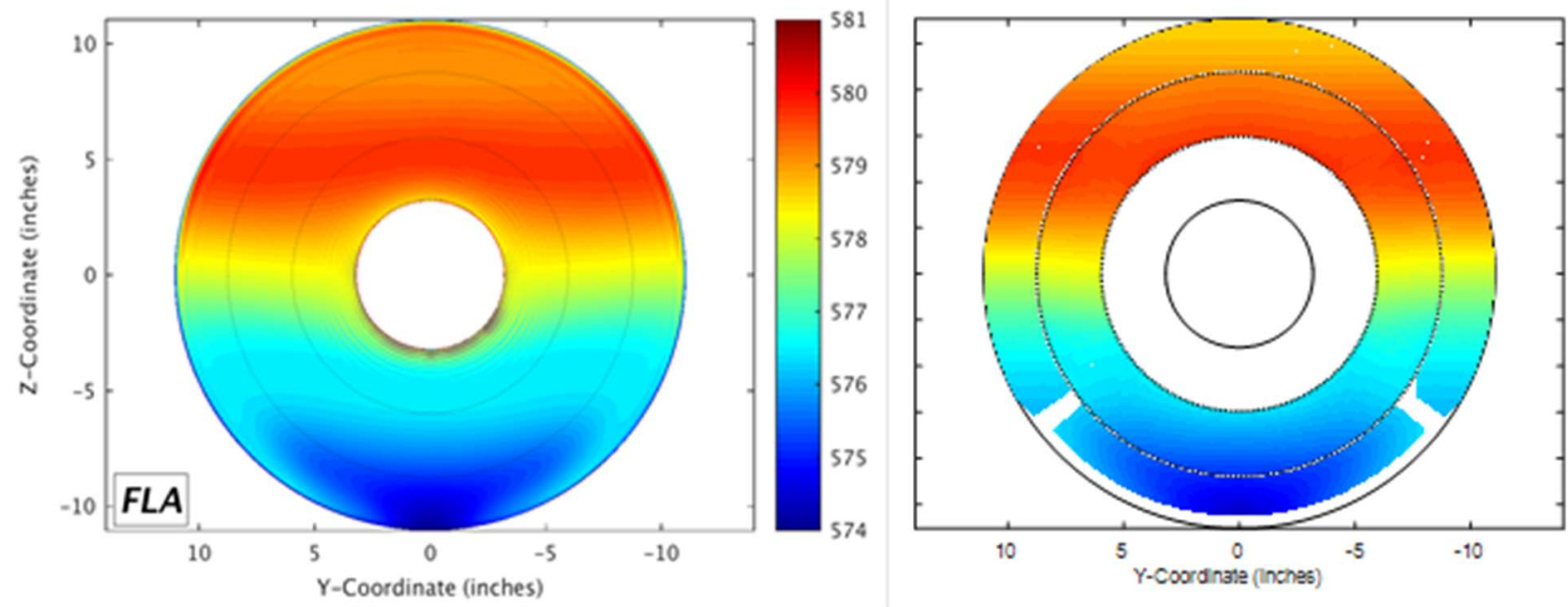

Figure 14. Full CFD Solution and Reconstruction from Sampled Data

Once again, it is important to understand whether the actual measured data is sufficiently well behaved to permit application of this technique to infer the temperatures in the outer annular region of the AIP. In Figure 15 it can be seen that there is more scatter than with the CFD and that there are two lines, indicating that the experiment has some left-right asymmetry. The curve fit shown by the green line is entirely based on the outer ring of total temperature. The outer nacelle probes do not seem to track the same profile as the AIP probes and were thus eliminated from the fit. The extent of the green curve shows the vertical extent of the range over which this fit will be employed. It does not extend to the bottom of the AIP because that has already been filled in using the Tt-Pt relationship previously described.

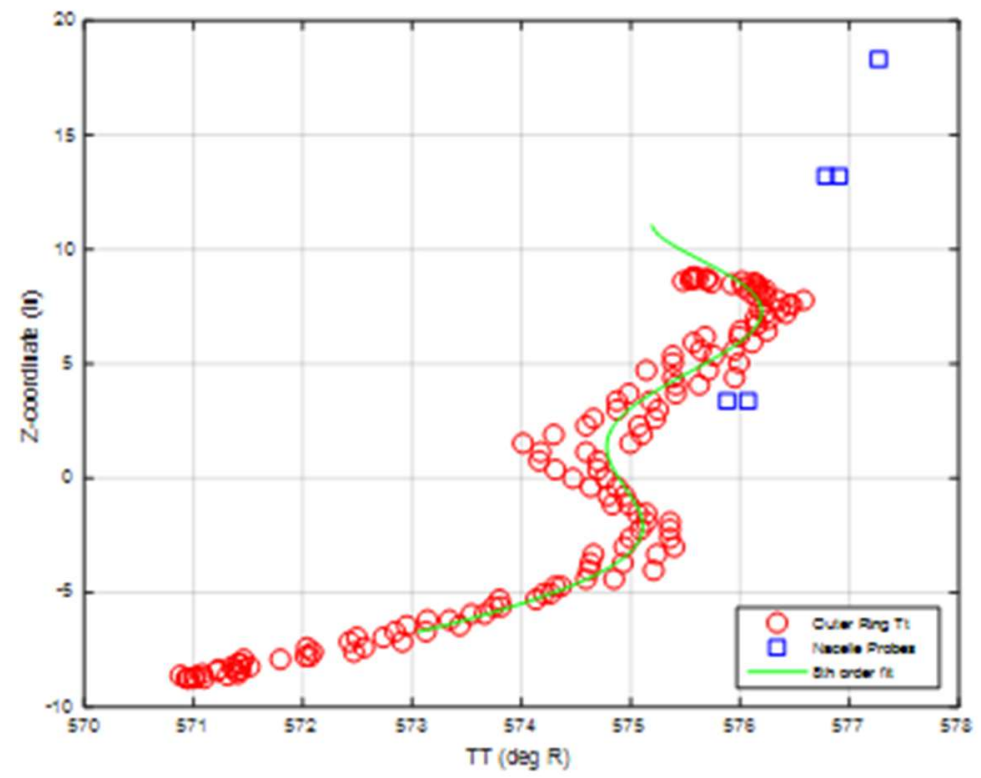

Figure 15. Curve Fit to Experimental Data Inside and Outside Nacelle 
The inner annular region of the AIP is filled in by interpolating the inner ring of AIP total temperature probes. (See Figure 16 and Figure 18.) The left panel shows the sampled CFD and the right shows the experiment.
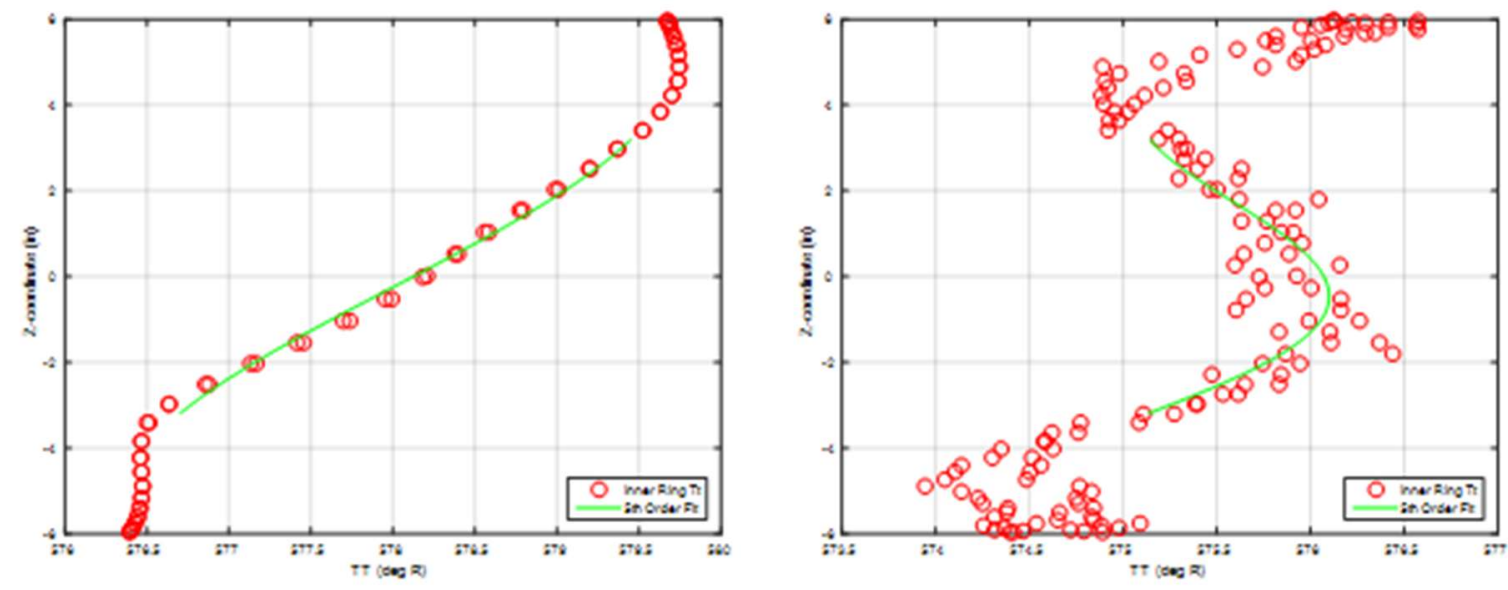

Figure 16. Curve Fit to Inner Ring of AIP Total Temperature Probes

It is obvious that the simplistic profile modeled in the CFD is not an accurate duplicate of the experiment; however, there is still a clear relationship that permits using the interpolating function to fill in the missing inner annular region. The total temperature contours on the left in Figure 17 are the full CFD solution while the contours on the right are reconstructed from the sampled CFD, which approximates the experimental measurements, by the previously discussed techniques. The mass-averaged total temperature determined from the sampled data is a remarkable match to that obtained from the full CFD solution. This is at least partly due to the fact that there is only a 7 degree $\mathrm{R}$ variation over the face of the AIP.

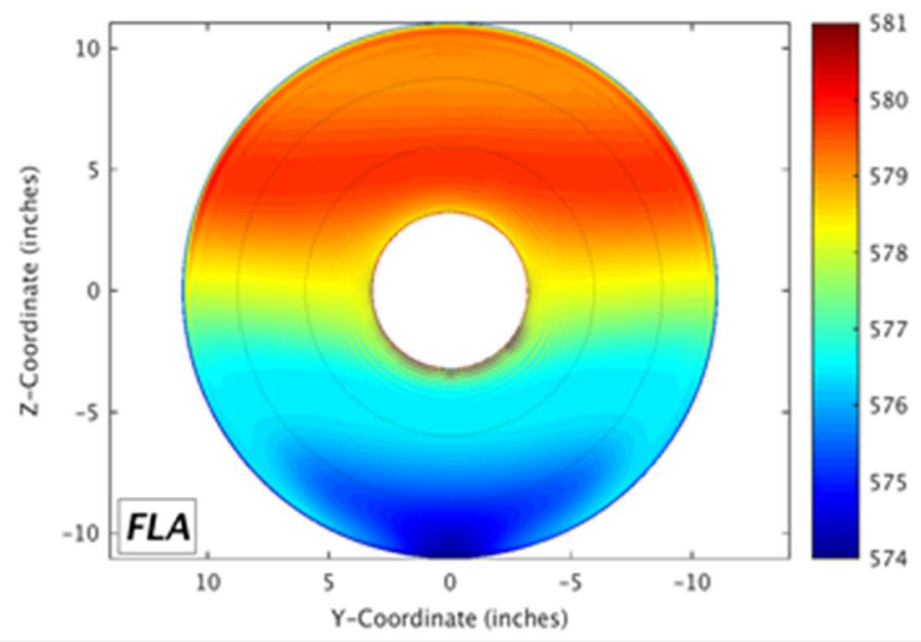

$T_{t}(M A)=577.95^{\circ} \mathrm{R}$

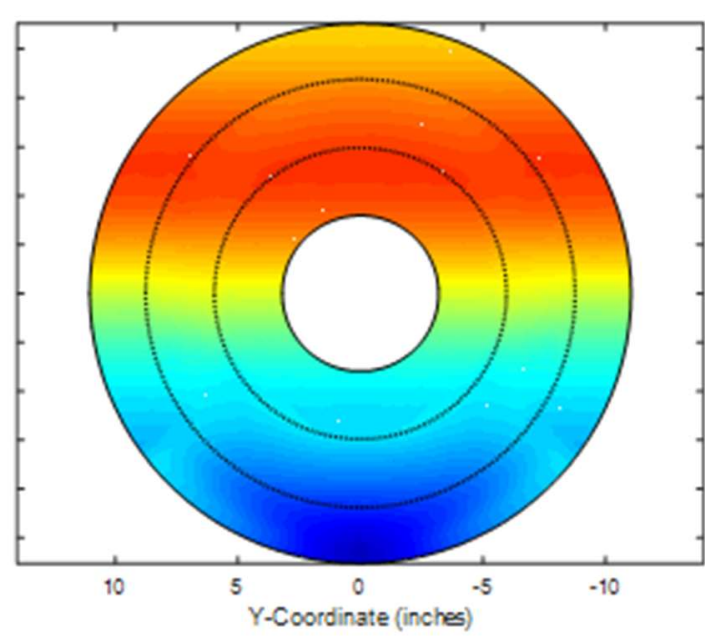

$T_{t}(M A)=577.92^{\circ} R$

Figure 17. Reconstruction of Total Temperature at AIP Compared to Full CFD Solution

It is not possible to prove that the techniques that have been demonstrated will accurately predict the "missing" experimental data. Comparison of the sampled CFD results with the full CFD solution strongly suggests that the interpolation techniques work, and the basic character of the experimental measured data is reflected in the CFD 
solution with the upstream $\mathrm{P}_{\mathrm{T}}$ and $\mathrm{T}_{\mathrm{T}}$ profiles. The techniques described here were applied to the experimental data obtained by rotating the AIP probe array to compute the mass-averaged total temperature at the AIP for the Aerodynamic Design Point (ADP) displayed in Figure 18. To be clear, the CFD was used to validate the methodology, but the results presented in Figure 18 come entirely from the experimental measurements. The same techniques were used at other flow conditions to calculate the efficiency numbers presented in Ref. [8] and Ref. [9]. These same results were used to generate the pressure ratio and efficiency plots previously shown in Figure 6and Figure 7.

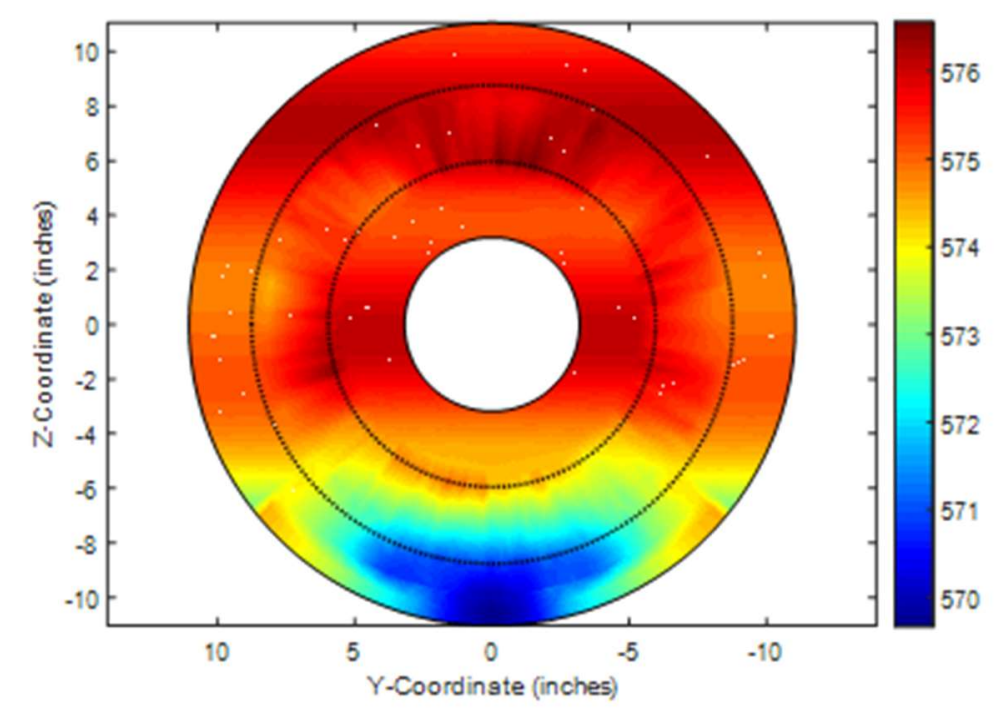

Figure 18. Total Temperatures at AIP from Experimental Measurements for the ADP

\section{Conclusion}

The experiment was run in multiple phases because the blockage that would have resulted from installing all the rakes and probes simultaneously was unacceptable. The flow at the fan face was characterized by installing a set of rotating rakes immediately upstream of the fan (Phase I). The ratio of the mass-flow averaged total pressure at the fan face (i.e. at the AIP) as measured by these rakes to the free stream total pressure (outside the generated boundary layer profile) was found to be a linear function of the corrected flow through the rig as measured by the rakes at the EGV exit. Similarly, the ratio of the mass flow averaged total temperature at the fan face to an upstream reference temperature was found to be a linear function of that same corrected flow. The blockage of these rakes compromised the accuracy of the total pressure and total temperature measurements at the EGV exit; therefore, a separate set of measurements was made with the AIP rakes removed (Phase III). The stage performance, characterized by stage pressure rise and efficiency, was calculated by computing the mass-flow averaged total pressure and total temperature at the EGV exit and the corrected weight flow from the Phase III measurements and combining those parameters with AIP parameters computed from curves fits of the Phase I measurements. The result effectively models the performance of the stage with no inlet rakes installed.

During the testing, it was discovered that there was a distortion in total temperature in the inlet flow which did not permit the assumption of uniform upstream total temperature to be used in computing stage performance. A CFD model of the experiment was modified to insert a similar total temperature distortion in order to evaluate a means of inferring the complete total temperature field at the fan face so that an accurate estimate of the mass-flow averaged total temperature could be computed. Curve fits to various subsets of the measured total temperatures were used to extend the two rings of measured temperatures to fill the fan face. The manner in which these fits were constructed was based on a limited knowledge of the physics of the flow field guided by the CFD solution. 


\section{Acknowledgements}

This effort was supported by NASA's Advanced Air Transport Technology Project. The authors gratefully acknowledge the support of the many additional people at UTRC who made this work possible, including Razvan Florea for his initiation of the program and initial inlet design and analysis work, Om Sharma for his continuous consultation throughout the project, and Eric Gray, Lawrence Binek and Matthew Kennedy for their detailed mechanical analysis. Major support was provided by the staff of the NASA 8'x6' Supersonic Wind Tunnel facility, and NASA's design and manufacturing teams.

\section{References}

[1] Smith, L. H., Jr., Wake Ingestion Propulsion Benefit. AIAA Journal of Propulsion and Power, Vol. 9, No. 1, pp. 74 - 82, January - February 1993.

[2] Daggett, D., Kawai, R., and Friedman, D., Blended Wing Body Systems Studies: Boundary Layer Ingestion Inlets with Active Flow Control, NASA CR 2003-212670, December 2003.

[3] Kawai, R. T., Friedman, D. L., and Serrano, L., Blended Wing Body (BWB) Boundary Layer Ingestion (BLI) Inlet Configuration and System Studies. NASA CR-2006-214534, December 2006.

[4] Hardin, L. W., Tillman, T. G., Sharma, O. P., Berton, J, and Arend, D. J., Aircraft System Study of Boundary Layer Ingesting Propulsion, AIAA Joint Propulsion Conference, July-August 2012.

[5] Florea, R.V., Matalantis, C., Hardin, L.W., Stucky, M., and Shabbir, A., "Parametric Analysis and Design for Embedded Engine Inlets," 48th AIAA/ASME/SAE/ASEE Joint Propulsion Conference \& Exhibit, AIAA 2012-3994, July 2012.

[6] Hirt, S.M., Arend, D.J. and Wolter, J.D., "Development of a Flow Field for Testing a Boundary-Layer-Ingesting Propulsor," Paper Submitted for Presentation at the AIAA Propulsion and Energy Forum, Atlanta, Georgia, July 10 - 12, 2017.

[7] Wolter, J.D., Arend, D.J., Hirt, S.M., and Gazzaniga, J.A., "Development of a Rotating Rake Array for Turbomachinery Measurements," Paper Submitted for Presentation at the AIAA Propulsion and Energy Forum, Atlanta, Georgia, July 10 - 12 , 2017.

[8] Arend, D.J., Wolter, J.D., Hirt, S.M., Provenza, A.J., Gazzaniga, J.A., Cousins, W.T., Hardin, L.W., and Sharma, O.P., "Experimental Evaluation of an Embedded Bountary Layer Ingesting Propulsor for Highly Efficient Subsonic Cruise Aircraft," Paper Submitted for Presentation at the AIAA Propulsion and Energy Forum, Atlanta, Georgia, July 10 - $12,2017$.

[9] Cousins, W.T., Voytovych, D., Tillman, T.G., and Gray, E., "Design of a Distortion-Tolerant Fan for a Boundary-Layer Ingesting Embedded Engine Application,” AIAA-2017-5042. 